\title{
Logical Modelling of Regulatory Networks, Methods and Applications
}

\author{
Claudine Chaouiya · Elisabeth Remy
}

Published online: 31 May 2013

(C) Society for Mathematical Biology 2013

\begin{abstract}
About 40 years ago, seminal work by S. Kauffman (1969) and R. Thomas (1973) paved the way to the establishment of a coarse-grained, "logical" modelling of gene regulatory networks. This gave rise to an increasingly active field of research, which ranges from theoretical studies to models of networks controlling a variety of cellular processes (Bornholdt 2008; Glass and Siegelmann 2010). Briefly, in these models, genes (or regulatory components) are assigned discrete values that account for their functional levels of expression (or activity). A regulatory function defines the evolution of the gene level, depending on the levels of its regulators. This abstracted representation of molecular mechanisms is very convenient for handling large networks for which precise kinetic data are lacking.

Within the logical framework, one can distinguish several approaches that differ in the way of defining a model and its behaviour. In random Boolean networks, first introduced by S. Kauffman, the components are randomly assigned a set of regulators and a regulatory function that drives their evolution, depending on these regulators (Kauffman 1969). In threshold Boolean networks, the function is derived from a given regulatory structure as a sum of the input signals, possibly considering a threshold (Bornholdt 2008; Li et al. 2004). In the generalised logical approach introduced by R. Thomas, the logical functions are general, but are constrained by the regulatory graph. Whenever necessary or useful, the formalism supports multi-valued variables
\end{abstract}

C. Chaouiya ( $\varangle)$

Instituto Gulbenkian de Ciência, Rua da Quinta Grande 6, Apartado 14, Oeiras, Lisboa 2780 156,

Portugal

e-mail: chaouiya@igc.gulbenkian.pt

E. Remy

Institut de Mathématiques de Luminy, Campus de Luminy, Case 907, 13288 Marseille cedex 9, France 
(Thomas and D'Ari 1990). More recently, logical interaction hypergraphs were proposed as an alternate representation (Klamt et al. 2006). Referring to the different approaches to define a model, it is worth noting that while a set of (Boolean) regulatory functions uniquely defines a regulatory graph, there may be several sets of functions compatible with a regulatory structure (Remy and Ruet 2008). Finally, it is worth mentioning attempts to overcome the determinism of regulatory functions in Boolean networks and to cope with uncertainty (e.g. Shmulevich et al. 2002).

In these discrete systems, elementary events are defined by updates of gene levels, governed by their regulatory functions. The dynamics of such systems are conveniently represented by state transition graphs (or diagrams). Diverse updating schemes have been proposed, which have an impact on the structure and properties of the resulting dynamics. The sole invariants are the stable states and the attracting elementary cycles in which each transition involves a unique component updating.

In the synchronous scheme (Kauffman 1993), updates take place simultaneously, assuming that all the response times associated with the updating component processes are similar. The dynamics are thus deterministic, each state having a unique successor (image). The state transition graph is composed of linear chains leading to attractors that are defined by stable states or elementary cycles. Other deterministic schemes can be defined, ranging from the synchronous (parallel) update, to the sequential update, in which components are successively updated following a given order, or else block-sequential schemes that define blocks of components that are sequentially updated, while components are synchronously updated within the blocks (e.g. Robert 1986; Demongeot et al. 2008).

The asynchronous updating as considered by R. Thomas decouples these updates, assuming that delays associated with actual changes of component levels differ (Thomas 1991). This turns the system non-deterministic: states may have several potential successors and the structure of the state transition graph may be highly complex. Alternatively, one can consider, for example, random asynchronous schemes (Chaves et al. 2005; Stoll et al. 2012), time delays (Siebert and Bockmayr 2006) or priority classes (Fauré et al. 2006).

A wide range of studies focus on the characterisation of the dynamics of generic logical models under specific updating schemes and specific classes of regulatory functions. These properties mainly relate to numbers of stable states, existence of cyclical attractors and also to basins of attraction. Regulatory circuits are known to play a crucial role in the dynamics of molecular networks (see Thieffry 2007 and references therein). More specifically, positive circuits are required for multi-stability, whereas stable oscillations originate from negative circuits. Hence, circuit analysis provides a valuable approach to relate a model with its dynamical properties (number and nature of its attractors).

A number of theoretical studies characterise the relationships between Boolean and continuous models (Glass and Kauffman 1973; Wilds and Glass 2009; Wittmann et al. 2009; Jamshidi et al. 2012). Indeed, while different mathematical frameworks are considered to model molecular networks (de Jong 2002), it is important to understand, for example, how properties of a given model translate to another framework. Moreover, coarse-grained discrete models can be used as a first step towards more quantitative, continuous models, which supposes a proper method for this conversion. 
In this context, piecewise linear differential models have gathered a particular interest because of their proximity to logical models (e.g. Glass and Kauffman 1973; Snoussi 1989; Chaves et al. 2010). In particular, qualitative simulations of such models, using constraints defined as inequalities between parameters, can also be represented by state transition graphs (Edwards et al. 2001; De Jong et al. 2004). Finally, methods are proposed to derive continuous models from Boolean ones (Wittmann et al. 2009; Mendoza and Xenarios 2006).

In terms of applicability, logical modelling (including all variations mentioned above) has been used to represent and analyse a wide range of molecular networks involved in the control of cellular processes (differentiation, proliferation, apoptosis), in different organisms, from Drosophila development (e.g. Albert and Othmer 2003; Chaves et al. 2005; González et al. 2006; Sánchez et al. 2008), to eukaryotic cell cycle (see Fauré and Thieffry 2009 for a survey). Immune response has also been tackled with logical modelling, from the early work of Kaufman et al. (1999), to more recent modelling efforts to account for $\mathrm{T}$ cell activation, differentiation and survival (e.g. Mendoza and Xenarios 2006; Saez-Rodriguez et al. 2007; Naldi et al. 2010; Saadatpour et al. 2011).

This brief overview does not do justice to the many contributors to this field; it only aims to illustrate the range of questions that have been addressed. The contributions to this special issue reflect the diversity of research studies related to the logical modelling. Importantly, new methodological needs arise when dealing with biological case studies, as illustrated by the last two articles.

The first three contributions relate to asynchronous logical models. We are fortunate to have a contribution from R. Thomas, who clarifies the role of the logical parameters (i.e. the regulatory functions) in defining all the concurrent trajectories in contrast to delay specifications that would select among these behaviours.

J-P. Comet et al. provide us with an updated discussion on the capacity of regulatory circuits in generating specific dynamical properties. Focussing on different notions of circuit functionality, they revisit and clarify these key properties.

Next, Lorenz et al. aim at reducing the complexity of the analysis of large, asynchronous state transition graphs by considering subgraphs derived from extremal states. Moreover, the authors investigate an alternate reduction by considering multivalued models amenable to Boolean representation.

Goles et al. consider threshold Boolean networks and delineate a method to assess the conservation of some properties of a model (in terms of attractors and their basins of attraction) when varying the deterministic update schemes. To illustrate their approach, the authors rely on two published models of the yeast cell cycle.

Chaves et al. propose to use the parameters of their piecewise affine differential model to define probabilities of transition in the directed graph representing the dynamics. This provides a valuable tool to further analyse the behaviour of the system under study, focussing on reachability and existence of periodic orbits.

Large-scale, comprehensive networks of interest can be handled thanks to the Boolean abstraction. With their comprehensive model of the influenza-host interactions during infection, Madrahimov et al. demonstrate this capability. Here, the Boolean model is synchronously updated and, following the approach introduced in Helikar et al. (2008), simulation specification allows one to define for each external 
signal, the probability that its state is 'ON' at each time step. Then a series of simulations is performed, leading to semi-quantitative representations of the activity levels of some molecular species.

To properly account for lymphocyte populations, one has to adopt a more sophisticated modelling approach as in Mendoza's contribution, which presents an original multilevel modelling of Th cell differentiation. This model takes into account both intra-cellular molecular networks and cell interactions. First, the author relies on a method previously presented in Mendoza and Xenarios (2006), which transforms a Boolean model with specific regulatory functions (a node is activated whenever at least one of its activators and none of its repressors are present) into a continuous model. The whole model of a virtual culture is then defined by a hundred of instances of the same cellular network (contained by each cell), together with pools of secreted cytokines through which cells interact.

Acknowledgements This special issue stems from a French-speaking workshop organised in Rabat, Morocco in April 2011. This one-week meeting was dedicated to the logical modelling of regulatory networks. We express our gratitude to El Houssine Snoussi from Mohammed V University for his precious help in organising this event, and we warmly thank the 21 participants for their involvement. We would like also to thank all the contributors to this special issue as well as the anonymous reviewers.

\section{References}

Albert, R., \& Othmer, H. G. (2003). The topology of the regulatory interactions predicts the expression pattern of the segment polarity genes in drosophila melanogaster. J. Theor. Biol., 223(1), 1-18.

Bornholdt, S. (2008). Boolean network models of cellular regulation: prospects and limitations. J. R. Soc. Interface, 5(Suppl 1), S85-S94.

Chaves, M., Albert, R., \& Sontag, E. D. (2005). Robustness and fragility of boolean models for genetic regulatory networks. J. Theor. Biol., 235(3), 431-449.

Chaves, M., Tournier, L., \& Gouzé, J.-L. (2010). Comparing boolean and piecewise affine differential models for genetic networks. Acta Biotheor., 58(2-3), 217-232.

de Jong, H. (2002). Modeling and simulation of genetic regulatory systems: a literature review. J. Comput. Biol., 1(9), 67-103.

De Jong, H., Gouzé, J.-L., Hernandez, C., Page, M., Sari, T., \& Geiselmann, J. (2004). Qualitative simulation of genetic regulatory networks using piecewise-linear models. Bull. Math. Biol., 66(2), 301-340.

Demongeot, J., Elena, A., \& Sené, S. (2008). Robustness in regulatory networks: a multi-disciplinary approach. Acta Biotheor, 56(1-2), 27-49.

Edwards, R., Siegelmann, H. T., Aziza, K., \& Glass, L. (2001). Symbolic dynamics and computation in model gene networks. Chaos, 11(1), 160-169.

Fauré, A., Naldi, A., Chaouiya, C., \& Thieffry, D. (2006). Dynamical analysis of a generic boolean model for the control of the mammalian cell cycle. Bioinformatics, 22(14), e124-e131.

Fauré, A., \& Thieffry, D. (2009). Logical modelling of cell cycle control in eukaryotes: a comparative study. Mol. BioSyst., 5(12), 1569-1581.

Glass, L., \& Kauffman, S. (1973). The logical analysis of continuous, non-linear biochemical control networks. J. Theor. Biol., 39(1), 103-129.

Glass, L., \& Siegelmann, H. T. (2010). Logical and symbolic analysis of robust biological dynamics. Curr. Opin. Genet. Dev., 20(6), 644-649.

González, A., Chaouiya, C., \& Thieffry, D. (2006). Dynamical analysis of the regulatory network defining the dorsal-ventral boundary of the drosophila wing imaginal disk. Genetics, 174, 1625-1634.

Helikar, T., Konvalina, J., Heidel, J., \& Rogers, J. A. (2008). Emergent decision-making in biological signal transduction networks. Proc. Natl. Acad. Sci. USA, 105(6), 1913-1918.

Jamshidi, S., Siebert, H., \& Bockmayr, A. (2012). Comparing discrete and piecewise affine differential equation models of gene regulatory networks. Lect. Notes Comput. Sci., 7223, 17-24. 
Kauffman, S. (1969). Metabolic stability and epigenesis in randomly constructed genetics nets. J. Theor. Biol., 22, 437-467.

Kauffman, S. A. (1993). The origins of order: self-organization and selection in evolution. New York: Oxford University Press.

Kaufman, M., Andris, F., \& Leo, O. (1999). A logical analysis of T cell activation and energy. Proc. Natl. Acad. Sci. USA, 96(7), 3894-3899.

Klamt, S., Saez-Rodriguez, J., Lindquist, J., Simeoni, L., \& Gilles, E. (2006). A methodology for the structural and functional analysis of signaling and regulatory networks. BMC Bioinform., 7, 56.

Li, F., Long, T., Lu, Y., Ouyang, Q., \& Tang, C. (2004). The yeast cell-cycle network is robustly designed. Proc. Natl. Acad. Sci. USA, 101(14), 4781-4786.

Mendoza, L., \& Xenarios, I. (2006). A method for the generation of standardized qualitative dynamical systems of regulatory networks. Theor. Biol. Med. Model., 3, 13.

Naldi, A., Carneiro, J., Chaouiya, C., \& Thieffry, D. (2010). Diversity and plasticity of Th cell types predicted from regulatory network modelling. PLoS Comput. Biol., 6, e1000912.

Remy, E., \& Ruet, P. (2008). From minimal signed circuits to the dynamics of boolean regulatory networks. Bioinformatics, 24(16), i220-i226.

Robert, F. (1986). Discrete iterations: a metric study (Vol. 6). Berlin: Springer.

Saadatpour, A., Wang, R.-S., Liao, A., Liu, X., Loughran, T. P., Albert, I., \& Albert, R. (2011). Dynamical and structural analysis of a $\mathrm{T}$ cell survival network identifies novel candidate therapeutic targets for large granular lymphocyte leukemia. PLoS Comput. Biol., 7(11), e1002267.

Saez-Rodriguez, J., Simeoni, L., Lindquist, J., Hemenway, R., Bommhardt, U., Arndt, B., Haus, U.-U., Weismantel, R., Gilles, E., Klamt, S., \& Schraven, B. (2007). A logical model provides insights into T cell receptor signaling. PLoS Comput. Biol., 3(8), e163.

Sánchez, L., Chaouiya, C., \& Thieffry, D. (2008). Segmenting the fly embryo: a logical analysis of the segment polarity cross-regulatory module. Int. J. Dev. Biol., 52(8), 1059-1075.

Shmulevich, I., Dougherty, E. R., Kim, S., \& Zhang, W. (2002). Probabilistic boolean networks: a rulebased uncertainty model for gene regulatory networks. Bioinformatics, 18(2), 261-274.

Siebert, H., \& Bockmayr, A. (2006). Incorporating time delays into the logical analysis of gene regulatory networks. Lect. Notes Comput. Sci., 4210, 169-183.

Snoussi, E. H. (1989). Qualitative dynamics of piecewise-linear differential equations: a discrete mapping approach. Dyn. Stab. Syst., 4(3-4), 565-583.

Stoll, G., Viara, E., Barillot, E., \& Calzone, L. (2012). Continuous time boolean modeling for biological signaling: application of Gillespie algorithm. BMC Syst. Biol., 6, 116.

Thieffry, D. (2007). Dynamical roles of biological regulatory circuits. Brief. Bioinform., 8(4), 220-225.

Thomas, R. (1973). Boolean formalisation of genetic control circuits. J. Theor. Biol., 42, 565-583.

Thomas, R. (1991). Regulatory networks seen as asynchronous automata: a logical description. J. Theor. Biol., 153, 1-23.

Thomas, R., \& D’Ari, R. (1990). Biological feedback. Boca Raton: CRC Press.

Wilds, R., \& Glass, L. (2009). Contrasting methods for symbolic analysis of biological regulatory networks. Phys. Rev. E, 80, 062902.

Wittmann, D. M., Krumsiek, J., Saez-Rodriguez, J., Lauffenburger, D. A., Klamt, S., \& Theis, F. J. (2009). Transforming boolean models to continuous models: methodology and application to T-cell receptor signaling. BMC Syst. Biol., 3, 98. 